\title{
Four-particle formalism of the CDW method in two-electron charge-exchange reactions
}

\author{
V.Yu. Lazur ${ }^{1 *}$, V.V. Aleksiy ${ }^{1}$, S.I. Myhalyna ${ }^{1}$, M. Hnatič ${ }^{2,3}$ \\ ${ }^{1}$ Uzhhorod National University, \\ 54, Voloshina str., 88000 Uzhgorod, Ukraine \\ ${ }^{2}$ Institute of Experimental Physics, Slovak Academy of Sciences \\ 47, Watsonova str., 04001 Košice, Slovakia \\ ${ }^{3}$ P.J. Šafarik University, \\ Park Angelinum 9, Košice, Slovakia \\ *E-mail: volodymyr.lazur@uzhnu.edu.ua
}

\begin{abstract}
The progress in solution of problems involving non-relativistic fast ion-atom collisions with two actively participating electrons has been reviewed. Leading quantum mechanical methods have been analyzed in the framework of four-body scattering theory. A simple formalism has been described on the basis of the Dodd-Greider integral equations for a four-particle system, which were used to analyze the two-electron charge-exchange process at medium and high velocities of relative motion of particles. An important feature of the developed four-particle continuum distorted waves (CDW) method is the consistent preservation of the proper asymptotic limits of the wave functions of a colliding system in the entrance and exit channels of the reaction, which takes into account the long-range nature of Coulomb interactions. The amplitude of the two-electron charge-exchange reaction has been calculated in the approximation of the mechanism of simultaneous capture by an incident particle of two target electrons. The calculations using the presented theory have been made on the example of a two-electron capture reaction in high-energy $\mathrm{He}^{2+}+\mathrm{He}$ collisions.
\end{abstract}

Keywords: Coulomb interaction, two-electron charge-exchange, ion-atom collisions, fourparticle formalism.

https://doi.org/10.15407/spqeo23.02.119

PACS 03.65.-w, 34.50.-s, 34.70.+e, 34.80.Dp, 31.15.-p

Manuscript received 18.02.20; revised version received 17.03.20; accepted for publication 10.06.20; published online 12.06.20.

\section{Introduction}

Due to the rapid development of computing technology in the recent twenty years, the increasing interest of physics-theorists is paying attention to atomic collisions, in particular, one- and two-electron processes in the field of intermediate and high energies [1-7]. The choice of two-electron processes as a research object is also conditioned by the importance of multidisciplinary applications of high-energy ion-atom collisions, ranging from fusion to medical accelerators for radiotherapy [8, 9]. A correct description of these collision processes requires the solution of a four-body problem with four active particles, namely: two nuclei and two electrons. Thus, the details of transfer of two electrons from the bound state of the target to the bound state of the projectile particle are sensitive to static electron-electron correlation in the initial and final states, as well as to the dynamic correlation during the collision.
Let's consider the problems of theoretical description of the processes including two-electron capture

$$
A^{Z_{\alpha}+}+B \rightarrow A^{\left(Z_{\alpha}-2\right)+}+B^{2+}
$$

in the region of intermediate and high velocities of collision of $A^{Z_{\alpha}{ }^{+}}$ions with $B$ atoms. The cross-sections of two-electron processes (1) are quite large, and therefore the contribution of these processes of stripping the atoms during the ion collisions should be taken into account along with one-electron ionization and oneelectron charge exchange, especially in the region of intermediate collision velocities where charge-exchange and ionization cross-sections are values of one order.

The asymptotic (at large internuclear distance) methods of atomic collision theory [4, 5, 10-13] developed in detail do not work in our case (1), since here, in contrast, small interatomic distances are significant. 
Physical features of processes of atomic particles scattering at intermediate energies are caused by strong distortion of electronic wave functions due to the presence of long-range Coulomb interaction between particles, as a result of which the real electron transitions from initial to final states are accompanied by a number of other, virtual, transitions. To describe these specific features of the Coulomb interaction, various approximate variants of Schrödinger formalism are used, the most popular of which is the continuum distorted waves (CDW) method [14]. Using this method in the problem of one-electron ionization and one-electron charge exchange leads to good agreement of the theory with the experimental data at high and average relative velocities. The successful application of the CDW method is largely due to the correct consideration of Coulomb asymptotic conditions in both reaction channels.

The advances made in the study of single-electron charge exchange and ionization processes based on the CDW method [1, 2] have prompted us to develop the Schrödinger formalism of the continuum distorted waves method to describe two-electron capture processes (1).

\section{General theory}

In the framework of nonrelativistic quantum mechanics, we consider collisions in a four-particle system $\alpha, \beta, \gamma_{1}$ and $\gamma_{2}$, in which three particles are coupled in the initial and final states, that is, they form a "compound" particle

$$
\alpha+\left(\beta ; \gamma_{1}, \gamma_{2}\right) \rightarrow\left(\alpha ; \gamma_{1}, \gamma_{2}\right)+\beta
$$

where the symbol $\left(\lambda ; \gamma_{1}, \gamma_{2}\right)$ denotes the corresponding compound particle $\left(\lambda=\alpha, \beta-\right.$ atomic nuclei and $\gamma_{1}, \gamma_{2}-$ electrons).

Without loss of generality, the particle spins can be not accounted, since the Coulomb effects that are interested by us are independent of the spins. Let introduce full Hamiltonian of the system $H_{0}+V$, where $H_{0}$ is the operator of the kinetic energy of four particles in the system of their center of mass,

$$
V=\sum_{k=1}^{2}\left(V_{\alpha, \gamma_{k}}+V_{\beta, \gamma_{k}}\right)+V_{\gamma_{1}, \gamma_{2}}+V_{\alpha, \beta}
$$

is the full interaction, $V_{\alpha, \gamma_{i}}$ - operator of pair interaction of the particles $\alpha$ and $\gamma_{1}$, etc.

We shall denote by $V_{\alpha}\left(V_{\beta}\right)$ the effective interaction potential that forms a compound particle in the initial (final) reaction channel (2); $H_{\alpha}=H_{0}+V_{\alpha}\left(H_{\beta}=H_{0}+V_{\beta}\right)$ is the Hamiltonian of the initial (final) channel; $G(W)=[W-H]^{-1}-$ the Green function of the Hamiltonian $H$. We also define the operator $v_{\lambda}=V-V_{\lambda}$ $(\lambda=\alpha, \beta)$.

The transition amplitude $T_{\alpha \beta}^{-}$from the channel " $\alpha$ " to channel " $\beta$ " in prior formalism is given in the standard form:

$$
\begin{aligned}
& T_{\alpha \beta}^{-}=\lim _{W \rightarrow E+i 0}\left\langle\Phi_{\beta}\left|v_{\alpha}+v_{\beta} G(W) v_{\alpha}\right| \Phi_{\alpha}\right\rangle \equiv \\
& \equiv\left\langle\Phi_{\beta}\left|U_{\alpha \beta}^{-}\right| \Phi_{\alpha}\right\rangle .
\end{aligned}
$$

Here, $U_{\alpha \beta}^{-}$is the transition operator from the channel " $\alpha$ " to channel " $\beta$ "; $\left\langle\Phi_{\beta}|,| \Phi_{\alpha}\right\rangle$ are the final and initial asymptotic states of a system, respectively, which are eigenfunctions of the operators $H_{\beta}, H_{\alpha}$ with the eigenvalues $\tilde{E}_{\beta}, \tilde{E}_{\alpha}$. On the energy surface $\tilde{E}_{\beta}=$ $=\tilde{E}_{\alpha}=E ; E$ is the total energy of a four-body system.

For the transition operator in a three-particle system, we can write the integral equation first obtained by Dodd and Greider [15]. Acting on the same scheme as in the three-body case [15], it is possible to write a similar equation also for a transition operator in a fourbody system. To this aim, we present the channel interaction $v_{\lambda}(\lambda=\alpha, \beta)$ in the form of the sum of two terms $v_{\lambda}=\left(v_{\lambda}-w_{\lambda}\right)+w_{\lambda}$, the explicit form of which is given below. Here, $w_{\alpha}$ and $w_{\beta}$ are the distorting potentials in the input and output channels of the reaction (2). Corresponding to these potentials, we shall introduce the Møller wave operators:

$$
\begin{aligned}
& \omega_{\alpha}^{+}=1+\left(E-H_{\alpha}-w_{\alpha}+i \varepsilon\right)^{-1} w_{\alpha}=1+g_{\alpha}^{+} w_{\alpha}, \\
& \omega_{\beta}^{-}=1+\left(E-H_{\beta}-w_{\beta}-i \varepsilon\right)^{-1} w_{\beta}=1+g_{\beta}^{-} w_{\beta},
\end{aligned}
$$

where $\varepsilon$ is an infinitely small positive number.

In the prior formalism of this theory, the potential $w_{\beta}$ is arbitrary, and the potential $w_{\alpha}$ should not lead to the reorganization of the $\beta$ channel, that is $\lim _{\varepsilon \rightarrow 0}\left\langle\Phi_{\beta}\left|\omega_{\alpha}^{+}\right| \Phi_{\alpha}\right\rangle=0$.

In accord with analogy with the three-body case [2], we introduce the auxiliary potential $v_{\chi}$, which corresponds to an intermediate virtual channel " $\chi$ " and the Green operator $g_{\chi}^{+}=\left(E-H+v_{\chi}+i \varepsilon\right)^{-1}$. Then the equation for a four-body transition operator with accounting the notations used looks like

$$
\begin{aligned}
& U_{\alpha \beta}^{-}=\omega_{\beta}^{-*}\left\{\left[1+\left(\mathrm{v}_{\beta}-w_{\beta}\right) g_{\chi}^{+}\right]\left(\mathrm{v}_{\alpha}-w_{\alpha}\right) \omega_{\alpha}^{+}+\right. \\
& \left.+\left(\mathrm{v}_{\beta}-w_{\beta}\right) g_{\chi}^{+} \mathrm{v}_{\chi} G_{\beta}^{+} U_{\alpha \beta}^{-}\right\} .
\end{aligned}
$$

So far, this is an exact equation. Now let's make an approximation for the transition operator $U_{\alpha \beta}^{-}$, namely, hold only the zero iteration in the right-hand side of the equation (7). As a result, we have the following representation of transition amplitude $T_{\alpha \beta}^{-}$

$$
\begin{aligned}
& T_{\alpha \beta}^{-}=\left\langle\Phi_{\beta}\left|\omega_{\beta}^{-*}\left[1+g_{\chi}^{+}\left(v_{\beta}-w_{\beta}\right)\right]\left(v_{\alpha}-w_{\alpha}\right) \omega_{\alpha}^{+}\right| \Phi_{\alpha}\right\rangle= \\
& =T_{\alpha \beta}^{-}(\mathrm{DWB})+\left\langle\Phi_{\beta}\left|\omega_{\beta}^{-*}\left[g_{\chi}^{+}\left(v_{\beta}-w_{\beta}\right)\right]\left(v_{\alpha}-w_{\alpha}\right) \omega_{\alpha}^{+}\right| \Phi_{\alpha}\right\rangle,
\end{aligned}
$$


where $T_{\alpha \beta}^{-}(\mathrm{DWB})=\left\langle\Phi_{\beta}\left|\omega_{\beta}^{-*}\left(v_{\alpha}-w_{\alpha}\right) \omega_{\alpha}^{+}\right| \Phi_{\alpha}\right\rangle \quad$ is the amplitude of the reaction (2) in the distorted wave Born approximation (DWB). The first term on the right-hand side of Eq. (8) corresponds to the direct electronic transitions from one atomic particle to another without additional re-scattering, while the second term describes two-step transitions of electrons through a continuous spectrum from a target atom to the states bound to the projectile particle.

\section{Amplitude of two-electron charge-exchange}

To describe the four-body system in the coordinate representation, we distinguish two standard sets of the reduced coordinates, $\vec{r}_{\alpha}, \vec{x}_{k}^{\prime}$ and $\vec{r}_{\beta}, \vec{s}_{k}^{\prime}(k=1,2)$. These values are expressed in the coordinates of the particles $\vec{r}_{i}$ and their masses $m_{i}(i=1,2,3,4)$ by formulas

$$
\begin{aligned}
& \vec{r}_{\alpha}=\vec{r}_{3}-\left(\vec{r}_{1}+\vec{r}_{2}+m_{\beta} \vec{r}_{4}\right) /\left(m_{\beta}+2\right), \\
& \vec{x}_{k}^{\prime}=\vec{r}_{k}-\left(m_{\beta} \vec{r}_{4}+\sum_{i=1}^{k-1} \vec{r}_{i}\right) /\left(m_{\beta}+k-1\right), \\
& \vec{r}_{\beta}=\vec{r}_{4}-\left(\vec{r}_{1}+\vec{r}_{2}+m_{\alpha} \vec{r}_{3}\right) /\left(m_{\alpha}+2\right), \\
& \vec{s}_{k}^{\prime}=\vec{r}_{k}-\left(m_{\alpha} \vec{r}_{3}+\sum_{i=1}^{k-1} \vec{r}_{i}\right) /\left(m_{\alpha}+k-1\right),
\end{aligned}
$$

where the indices 1, 2, 3, 4 number the particles $\gamma_{1}, \gamma_{2}$, $\alpha, \beta$, respectively, $m_{\alpha, \beta}=m_{3,4} / m$ and $m_{1}=m_{2}=m$.

Let introduce the radius-vectors $\vec{x}_{k}$ and $\vec{s}_{k}$ that determine the position of $k$-th electron $\left(\gamma_{k}\right)$ related with the $\beta$ and $\alpha$ nuclei, respectively; their difference $\vec{x}_{k}-\vec{s}_{k}=\vec{R}$ is the distance between the $\beta$ and $\alpha$ nuclei. In these notations, the channel interactions $v_{\alpha}$ and $v_{\beta}$ have the form

$$
\begin{aligned}
& v_{\alpha}=-\frac{Z_{\alpha}}{s_{1}}+\frac{Z_{\alpha} Z_{\beta}}{R}-\frac{Z_{\alpha}}{s_{2}}, \\
& v_{\beta}=-\frac{Z_{\beta}}{x_{1}}+\frac{Z_{\alpha} Z_{\beta}}{R}-\frac{Z_{\beta}}{x_{2}},
\end{aligned}
$$

where $Z_{\alpha}$ and $Z_{\beta}$ are the charges of nuclei $\alpha$ and $\beta$, respectively.

By definition, the Hamiltonian $H_{\alpha}\left(H_{\beta}\right)$ describes an asymptotic situation when the particle $\alpha(\beta)$ does not interact with anything, and the other three ones are in the bound state in the potential $V_{\alpha}\left(V_{\beta}\right)$. The eigenstates $\left|\Phi_{\alpha}\right\rangle\left(\left|\Phi_{\beta}\right\rangle\right)$ of the Hamiltonian $H_{\alpha}\left(H_{\beta}\right)$ are the product of the wave function $\varphi_{\alpha}\left(\vec{x}_{1}^{\prime}, \vec{x}_{2}^{\prime}\right)\left(\varphi_{\beta}\left(\vec{x}_{1}^{\prime}, \vec{x}_{2}^{\prime}\right)\right)$ of the bound state of the system $\left(\beta ; \gamma_{1}, \gamma_{2}\right)\left(\left(\alpha ; \gamma_{1}, \gamma_{2}\right)\right)$ and of a plane wave of the relative motion of particles in the initial (final) state:

$$
\begin{aligned}
& \Phi_{\alpha}=\varphi_{\alpha}\left(\vec{x}_{1}^{\prime}, \vec{x}_{2}^{\prime}\right) \exp \left(i \vec{k}_{\alpha} \vec{r}_{\alpha}\right), \\
& \Phi_{\beta}=\varphi_{\beta}\left(\vec{s}_{1}^{\prime}, \vec{s}_{2}^{\prime}\right) \exp \left(-i \vec{k}_{\beta} \vec{r}_{\beta}\right),
\end{aligned}
$$

where $\vec{k}_{\alpha}\left(\vec{k}_{\beta}\right)$ is the momentum of the incident (scattered) particle in the system of the mass center before (after) collision.

Let us now discuss the physical content of the operators included in the formula (8). From the formal point of view, the operator $\omega_{\alpha}^{+}\left(\omega_{\beta}^{-}\right)$can be considered as an operator that transforms the initial (final) asymptotic state of system $\left|\Phi_{\alpha}\right\rangle\left(\left|\Phi_{\beta}\right\rangle\right)$ into a distorted wave $\left|\chi_{\alpha}^{+}\right\rangle\left(\left|\chi_{\alpha}^{-}\right\rangle\right)$in the entrance (in the exit) reaction channel:

$\left|\chi_{\alpha}^{+}\right\rangle=\omega_{\alpha}^{+}\left|\Phi_{\alpha}\right\rangle$,

$\left|\chi_{\beta}^{-}\right\rangle=\omega_{\beta}^{-}\left|\Phi_{\beta}\right\rangle$.

Finally, $U_{\alpha}=v_{\alpha}-w_{\alpha}$ can be considered as an operator that causes the transition of the system from initial state $(\alpha)$ to the final one $(\beta)$.

We introduce the state vector $\left|\Psi_{\beta}^{-}\right\rangle$, defining it with the expression:

$\left|\Psi_{\beta}^{-}\right\rangle=\left[1+g_{\chi}^{+^{*}}\left(v_{\beta}-w_{\beta}\right)\right]\left|\chi_{\beta}^{-}\right\rangle$.

In notations (13)-(15), the transition amplitude (8) can be represented as follows:

$T_{\alpha \beta}^{-}=\left\langle\Psi_{\beta}^{-}\left|U_{\alpha}\right| \chi_{\alpha}^{+}\right\rangle$.

We obtain an explicit form of differential equations for the calculation of distortions in the initial and final channels of the reaction. Applying the operator $\left(E-H_{\alpha}-w_{\alpha}\right)$ to both parts of the equality (13) and directing $\varepsilon \rightarrow 0^{+}$, we obtain the following equation for the distorted wave in the entrance channel:

$$
\begin{aligned}
& \left(E-H_{\alpha}-w_{\alpha}\right)\left|\chi_{\alpha}^{+}\right\rangle=\left(E-H_{\alpha}\right)\left|\Phi_{\alpha}\right\rangle=0, \\
& E=E_{\alpha}+k_{\alpha}^{2} / 2 \mu_{\alpha} .
\end{aligned}
$$

Similar equations can be derived from (14) for the distorted wave in the exit channel:

$$
\begin{aligned}
& \left(E-H_{\beta}-w_{\beta}\right)\left|\chi_{\beta}^{-}\right\rangle=\left(E-H_{\beta}\right)\left|\Phi_{\beta}\right\rangle=0, \\
& E=E_{\beta}+k_{\beta}^{2} / 2 \mu_{\beta} .
\end{aligned}
$$

Here, $E_{\alpha}$ and $E_{\beta}$ are the energies of the bound states of the compound particles $\left(\beta ; \gamma_{1}, \gamma_{2}\right)$ and $\left(\alpha ; \gamma_{1}, \gamma_{2}\right)$; $\mu_{\alpha}=m_{\alpha}\left(m_{\beta}+2\right) / M, \mu_{\beta}=m_{\beta}\left(m_{\alpha}+2\right) / M$ are the reduced masses of the corresponding groups of particles and $M=m_{\alpha}+m_{\beta}+2$ is the total mass of the system.

Further derivations are based on one "technical" requirement that will help to find an explicit solution to the task under our consideration. In fact, this will be a special choice of the function $\chi_{\beta}^{-}$based on simple physical considerations. We will require that the solution $\chi_{\beta}^{-}$of Eq. (18) should be represented in a factorized form 
$\left|\chi_{\beta}^{-}\right\rangle=\left|\varphi_{\beta}\left(\vec{s}_{1}^{\prime}, \vec{s}_{2}^{\prime}\right) f\left(\vec{r}_{\beta}\right)\right\rangle$,

where the function $f\left(\vec{r}_{\beta}\right)$ describes the asymptotic motion of the bound system of three-particles $\left(\alpha ; \gamma_{1}, \gamma_{2}\right)$ in the Coulomb field that is created by the fourth particle $\beta$.

This requirement is in fact an additional assumption. The fact is that the representation for a multiparticle wave function $\chi_{\beta}^{-}$in the form (19) is applied if the relative velocity of heavy particles is higher than the orbital velocity of bound electron. However, if the collision velocity is small and the nucleus $\beta$ interacts with the particles $\alpha, \gamma_{1}, \gamma_{2}$ for a rather long time, then this factorization is hardly grounded.

The differential equation (18) must be supplemented with the boundary condition, which in this case has the following form:

$$
\begin{aligned}
& \chi_{\beta}^{-} \underset{r_{\beta} \rightarrow \infty}{\longrightarrow} \varphi_{\beta}\left(\vec{s}_{1}^{\prime}, \vec{s}_{2}^{\prime}\right) \times \\
& \times \exp \left\{-i \vec{k}_{\beta} \vec{r}_{\beta}-i \frac{Z_{\beta}\left(Z_{\alpha}-2\right)}{v^{\prime}} \ln \left(k_{\beta} r_{\beta}-\vec{k}_{\beta} \vec{r}_{\beta}\right)\right\}, \\
& \vec{v}^{\prime}=\vec{k}_{\beta} / k_{\beta} .
\end{aligned}
$$

The fulfillment of the conditions that were made for the function $\chi_{\beta}^{-}$is easily achieved by the appropriate choice of the distortion potential $w_{\beta}$ in Eq. (18). As $w_{\beta}$ one can choose, for example, the potential $w_{\beta}^{(0)}=Z_{\beta}\left(Z_{\alpha}-2\right) / r_{\beta}$, then the function $f\left(\vec{r}_{\beta}\right)$ can be expressed explicitly through the degenerate hypergeometric function. However, we will not present the appropriate formulas, since the explicit form of $f\left(\vec{r}_{\beta}\right)$ is not essential here.

Applying the operator $\left(E-H_{\alpha}+v_{\chi}^{*}\right)$ to both parts of the expression (15) and taking into account (18), we obtain (in the limit $\varepsilon \rightarrow 0^{+}$) the differential equation

$$
\left(E-H_{\alpha}+v_{\chi}^{*}\right)\left|\Psi_{\beta}^{-}\right\rangle=v_{\chi}^{*}\left|\chi_{\beta}^{-}\right\rangle \text {. }
$$

Since the solution of the inhomogeneous equation (21) with realistic local potential is very difficult to deal with, it is logical to replace this potential with an operator, choosing it so that

$v_{\chi}^{*}\left|\chi_{\beta}^{-}\right\rangle=0$,

and transform Eq. (21) to the form similar to (19),

$$
\left|\Psi_{\beta}^{-}\right\rangle=\left|\varphi_{\beta}\left(\vec{s}_{1}^{\prime}, \vec{s}_{2}^{\prime}\right) \mathfrak{I}^{-}\right\rangle,
$$

where the still unknown function $\mathfrak{I}^{-}$describes the distortion of the wave function $\varphi_{\beta}\left(\vec{s}_{1}^{\prime}, \vec{s}_{2}^{\prime}\right)$ of the bound state of the system $\left(\alpha ; \gamma_{1}, \gamma_{2}\right)$ due to its interaction with the nucleus $\beta$ in the output channel.

Due to the condition (22) the equation (21) is reduced to homogeneous one

$$
\left[E-H_{0}+\sum_{k=1}^{2}\left(\frac{Z_{\beta}}{x_{k}}+\frac{Z_{\alpha}}{s_{k}}\right)-\frac{Z_{\alpha} Z_{\beta}}{R}-\frac{1}{\left|\vec{s}_{1}-\vec{s}_{2}\right|}+v_{\chi}^{*}\right]\left|\Psi_{\beta}^{-}\right\rangle=0 .
$$

Using the above-defined two sets of relative variables, we write the operator $H_{0}$ in two equivalent forms

$$
H_{0}=-\frac{1}{2 \mu_{\alpha}} \Delta_{\vec{r}_{\alpha}}-\sum_{k=1}^{2} \frac{1}{2 \mu_{\beta k}} \Delta_{\vec{x}_{k}^{\prime}}=-\frac{1}{2 \mu_{\beta}} \Delta_{\vec{r}_{\beta}}-\sum_{k=1}^{2} \frac{1}{2 \mu_{\alpha k}} \Delta_{\vec{s}_{k}^{\prime}},
$$

where

$$
\mu_{\beta k}=\left(m_{\beta}+k-1\right) /\left(m_{\beta}+k\right), \mu_{\alpha k}=\left(m_{\alpha}+k-1\right) /\left(m_{\alpha}+k\right) .
$$

At first glance, it may seem that the problem of solving the equation (24) is generally trivial. In fact, it is not. Complications appear from the fact that the interaction potentials and the operator $H_{0}$ presenting in this equation depend on the various combinations of relative variables used in the task (for example, the Jacobi coordinates $\vec{r}_{\alpha}, \vec{x}_{k}^{\prime}$ or $\vec{r}_{\beta}, \vec{s}_{k}^{\prime}$, on which the operator $H_{0}$ depends, and the coordinates $\vec{x}_{k}^{\prime}, \vec{s}_{k}^{\prime}, R$, on which the interaction potentials depend).To avoid these difficulties, let us consider an approximate method of separating the variables in the equation (24), which is based on the explicit approximation that the masses of particles $\gamma_{1}, \gamma_{2}$ (electrons) are much smaller than the masses of the other two particles $\alpha$ and $\beta$ (atomic nuclei), that is $m_{1}=m_{2}<<m_{3,4}$. In this case, in the expressions (9) and (10) one can neglect by the terms containing the mass ratio $m_{\mathrm{\kappa}} / m_{3,4}(k=1,2)$, as a result the Jacobi coordinates $\vec{x}_{k}^{\prime}$, $\vec{r}_{\alpha}, \vec{s}_{k}^{\prime}$, and $\vec{r}_{\beta}$ are close to the coordinates $\vec{x}_{k}^{\prime}, \vec{R}, \vec{s}_{k}^{\prime}$, and $-\vec{R}$, respectively, that is

$\vec{x}_{k}^{\prime} \cong \vec{x}_{k}, \vec{s}_{k}^{\prime} \cong \vec{s}_{k}, \vec{r}_{\alpha}=\vec{R}, \vec{r}_{\beta}=-\vec{R}$.

Substituting the wave function $\Psi_{\beta}^{-}$in the form (23) into the expression (24), we obtain, with account of (18) and (26), the equation for $\mathfrak{I}^{-}$

$$
\begin{aligned}
& \varphi_{\beta}\left(E-E_{\beta}-H_{0}-v_{\beta}\right) \mathfrak{I}^{-}+ \\
& +\sum_{k=1,2} \frac{1}{\mu_{\alpha, \beta}} \vec{\nabla}_{\vec{s}_{k}^{\prime}} \varphi_{\beta} \vec{\nabla}_{\vec{s}_{k}^{\prime}} \mathfrak{I}^{-}+v_{\chi}^{*}\left(\varphi_{\beta} \mathfrak{J}^{-}\right)=0 .
\end{aligned}
$$

We need to solve this equation, accounting the additional conditions (22) mentioned above as well as boundary condition specifying the dynamics. As $v_{\chi}$ in the last equation we choose the operator $v_{\chi}^{(0)}$, action of which on the arbitrary function $\Psi$ of $\vec{r}_{\beta}$ and $\vec{s}_{k}^{\prime}(k=1,2)$ is described by the relation:

$v_{\chi}^{(0)} \Psi=-\sum_{k=1,2}\left(\mu_{\alpha k}\right)^{-1} \vec{\nabla}_{\vec{s}_{k}^{\prime}} \varphi_{\beta} \vec{\nabla}_{\vec{s}_{k}^{\prime}}\left[\Psi / \varphi_{\beta}\right]$ 
Substituting the expression (28) $v_{\chi}^{(0)}$ in (27) and accounting (26), we obtain the equation for $\mathfrak{I}^{-}$of the following form:

$$
\left[E-E_{\beta}+\frac{1}{2 \mu_{\alpha}} \Delta_{\vec{r}_{\alpha}}+\sum_{k=1,2}\left(\frac{1}{2 \mu_{\beta k}} \Delta_{\vec{x}_{k}^{\prime}}+\frac{Z_{\beta}}{x_{k}^{\prime}}\right)-\frac{Z_{\alpha} Z_{\beta}}{r_{\alpha}}\right] \mathfrak{J}^{-}=0 .
$$

In what follows, we search the solution of the equation (29), which at infinity has the form of a distorted plane wave with the unity amplitude

$$
\begin{aligned}
& \mathfrak{I}^{-} \underset{r_{\beta} \rightarrow \infty}{\longrightarrow} f\left(\vec{r}_{\beta}\right) \underset{r_{\beta} \rightarrow \infty}{\longrightarrow} \\
& \exp \left\{-i \vec{k}_{\beta} \vec{r}_{\beta}-i \frac{Z_{\beta}\left(Z_{\alpha}-2\right)}{v^{\prime}} \ln \left(k_{\beta} r_{\beta}-\vec{k}_{\beta} \vec{r}_{\beta}\right)\right\} .
\end{aligned}
$$

Solving the equation (29) with the method of variable separations, we find that

$$
\mathfrak{I}^{-}=C^{-} \Lambda^{(-)}\left(\vec{r}_{\alpha}\right) \prod_{k=1}^{2} \Lambda_{k}^{(-)}\left(\vec{x}_{k}^{\prime}\right), C^{-}=\text {const },
$$

where two-particle Coulomb distorting functions $\Lambda_{k}^{(-)}\left(\vec{x}_{k}^{\prime}\right)$ and $\Lambda^{(-)}\left(\vec{r}_{\alpha}\right)$ are described in the terms of degenerate hypergeometric functions with the relations

$$
\begin{aligned}
& \Lambda_{k}^{(-)}\left(\vec{x}_{k}^{\prime}\right)=N^{(+)}\left(v_{\beta k}^{\prime}\right) \exp \left(i \vec{q}_{k} \vec{x}_{k}^{\prime}\right) F\left(-i v_{\beta k}^{\prime}, 1,-i \vec{q}_{k} \xi_{k}\right), \\
& \Lambda^{(-)}\left(\vec{r}_{\alpha}\right)=N^{(-)}\left(v_{\alpha}^{\prime}\right) \exp \left(i \vec{q}_{\alpha} \vec{r}_{\alpha}\right) F\left(i v_{\alpha}^{\prime}, 1,-i q_{\alpha} \xi_{\alpha}\right) .
\end{aligned}
$$

Here, $N^{( \pm)}(v)=\Gamma(1 \pm i v) \exp ( \pm \pi v / 2)$ are the normalization coefficients; $\xi_{k}=x_{k}^{\prime}+\hat{q}_{k} x_{k}^{\prime}, \quad \xi_{\alpha}=x_{k}^{\prime}+\hat{q}_{\alpha} r_{\alpha}^{\prime}$ - two-particle parabolic variables; $\hat{q}_{k}$ and $\hat{q}_{\alpha}$ - unity vectors directed along the vectors $\vec{q}_{k}$ and $\vec{q}_{\alpha}$; $v_{\beta k}^{\prime}=Z_{\beta} \mu_{\beta k} / q_{k}, \quad v_{\alpha}^{\prime}=Z_{\alpha} Z_{\beta} \mu_{\alpha} / q_{\alpha} \quad-\quad$ characteristic Coulomb parameters. It follows from the law of energy conservation that variables $\vec{q}_{k}$ and $\vec{q}_{\alpha}$ must satisfy the equation

$$
E-E_{\beta}=\frac{k_{\beta}^{2}}{2 \mu_{\beta}}=\frac{q_{\alpha}^{2}}{2 \mu_{\alpha}}+\sum_{k=1,2} \frac{q_{k}^{2}}{2 \mu_{\beta} k} .
$$

Further, considering the asymptotic form of the function $F(a, b, x)$ at $x \rightarrow \infty$, from the asymptotic conditions of function matching (31) with the eikonal asymptotics (30) we find that

$$
\begin{aligned}
& \sum_{k=1,2} \vec{q}_{k} \vec{x}_{k}^{\prime}+\vec{q}_{\alpha} \vec{r}_{\alpha}=-\vec{k}_{\beta} \vec{r}_{\beta}, \\
& C^{-} \exp \left\{i \sum_{k=1,2} v_{\beta k}^{\prime} \ln \left(q_{k} \xi_{k}\right)-i v_{\alpha}^{\prime} \ln \left(q_{\alpha} \xi_{\alpha}\right)\right\} \underset{r_{\beta} \rightarrow \infty}{\longrightarrow} \\
& \exp \left\{i \frac{Z_{\beta}\left(Z_{\alpha}-2\right)}{v} \ln \left(k_{\beta} r_{\beta}-\vec{k}_{\beta} \vec{r}_{\beta}\right)\right\}
\end{aligned}
$$

We express $\vec{r}_{\beta}$ through the coordinates $\vec{r}_{\alpha}, \vec{x}_{1}^{\prime}$ and $\vec{x}_{2}^{\prime}$ by using the formula

$\vec{r}_{\beta}=-a_{2} \vec{r}_{\alpha}-\sum_{k=1,2} \frac{b_{k}}{\mu_{\beta}} \vec{x}_{k}^{\prime}$,

$a_{k}=m_{\alpha} /\left(m_{\alpha}+k\right)$,

$b_{k}=m_{\beta} /\left(m_{\beta}+k\right)$.

We now substitute (37) into the right-hand side of the equality (35) and equate the terms at $\vec{r}_{\alpha}, \vec{x}_{1}^{\prime}$ and $\vec{x}_{2}^{\prime}$ in both its parts. We obtain

$$
\begin{aligned}
& \vec{q}_{k}=\vec{k}_{\beta} b_{k} / \mu_{\beta} \equiv b_{k} \vec{v}^{\prime} \underset{m_{\beta} \rightarrow \infty}{\longrightarrow} \vec{v}^{\prime} \quad(k=1,2), \\
& \vec{q}_{\alpha}=a_{2} \vec{k}_{\beta} \underset{m_{\alpha} \rightarrow \infty}{\longrightarrow} \vec{k}_{\beta} .
\end{aligned}
$$

Accounting the asymptotic limits

$-i \frac{Z_{\beta}}{u^{\prime}} \ln \left(\frac{k_{\beta} r_{\beta}-\vec{k}_{\beta} \vec{r}_{\beta}}{u^{\prime} x_{k}^{\prime}+\vec{v}^{\prime} \vec{x}_{k}}\right) \underset{r_{\beta} \rightarrow \infty}{\longrightarrow} \ln \left(\mu_{\beta}^{-i Z_{\beta} / v^{\prime}}\right)$

$k=1,2$

from the condition (36), we find that $C^{-}=\mu_{\beta}^{2 i Z_{\beta} / v^{\prime}}$. It follows from the formulas (31) to (33) and (38) that the distortion function $\mathfrak{J}^{-}$in the output channel can be represented as

$$
\begin{aligned}
& \mathfrak{I}^{-}=\mu_{\beta}^{2 i v_{\beta}^{\prime}} N^{(-)}\left(v^{\prime}\right)\left[N^{(+)}\left(v_{\beta}^{\prime}\right)\right]^{2} \exp \left(-i \vec{k}_{\beta} \vec{r}_{\beta}\right) \times \\
& \times F\left(i v^{\prime}, 1,-i k_{\beta} r_{\alpha}-i \vec{k}_{\beta} \vec{r}_{\alpha}\right) \prod_{k=1}^{2} F\left(-i v_{\beta}^{\prime}, 1,-i v^{\prime} x_{k}^{\prime}-i \vec{v}^{\prime} x_{k}^{\prime}\right),
\end{aligned}
$$

where $v_{\beta}^{\prime}=Z_{\beta} / u^{\prime}, v=Z_{\alpha} Z_{\beta} / u^{\prime}$.

Therefore, the wave function of the finite state $\Psi_{\beta}^{-}$ (defined by the formulas (23), (40)) has been derived, which in the present task describes the scattering of the charged particle $\beta$ by the bound compound of three particles $\alpha, \gamma_{1}, \gamma_{2}$. The important thing is that the effects of pair Coulomb interactions in it manifested themselves in a multiplicative way. Further, we describe the wave function of the initial state. This function can be obtained by solving the differential equation (17). We complete the above equation with the boundary condition on infinity

$$
\begin{aligned}
& \chi_{\alpha}^{+} \underset{r_{\alpha} \rightarrow \infty}{\longrightarrow} \varphi_{\alpha}\left(\vec{x}_{1}^{\prime}, \vec{x}_{2}^{\prime}\right) \times \\
& \times \exp \left\{i \vec{k}_{\alpha} \vec{r}_{\alpha}+i \frac{Z_{\alpha}\left(Z_{\beta}-2\right)}{v} \ln \left(k_{\alpha} r_{\alpha}-\vec{k}_{\alpha} \vec{r}_{\alpha}\right)\right\}, \\
& \vec{v}=\vec{k}_{\alpha} / \mu_{\alpha} .
\end{aligned}
$$

We substitute the function in the form of a product

$\chi_{\alpha}^{+}=\varphi_{\alpha}\left(\vec{x}_{1}^{\prime}, \vec{x}_{2}^{\prime}\right) \mathfrak{I}^{+}$

into the equation (17) and, by making simple transformations, we obtain the equation for $\mathfrak{J}^{+}$: 
$\varphi_{\alpha}\left(E-E_{\alpha}-H_{0}-v_{\alpha}\right) \mathfrak{I}^{+}+$

$+\sum_{k=1,2}\left(\mu_{\beta k}\right)^{-1} \vec{\nabla}_{\vec{x}_{k}^{\prime}} \varphi_{\alpha} \vec{\nabla}_{\vec{x}_{k}^{\prime}} \mathfrak{I}+U_{\alpha}\left(\varphi_{\alpha} \mathfrak{I}^{+}\right)=0$.

In further calculations, we will need a specific kind of the operator $U_{\alpha}=\varphi_{\alpha}-w_{\alpha}$. Only the most general considerations can be given when choosing $U_{\alpha}$. First, it is clear that this operator must be such that the equation (43) has solutions in the class of special or elementary functions. It is necessary to watch that the wave function of the initial state $\chi_{\alpha}^{+}$has the correct asymptotic behavior (41) over long distances.

Second, the operator $U_{\alpha}$ must be selected so that the distortions in the entrance $\left(\mathfrak{I}^{+}\right)$and in the exit $\left(\mathfrak{I}^{-}\right)$ channels of the reaction (2) were treated equally. The fact is that asymmetric choice of $v_{\chi}$ and $U_{\alpha}$ in the equations (27) and (43) leads to asymmetric definition of distortions in the initial and final states, which contradicts the basic idea of the CDW method [14]. In addition, if some a priori information is known about the scattering wave functions $\Psi_{\beta}^{-}$and $\chi_{\alpha}^{+}$, then it can be also taken into account when choosing $v_{\chi}$ and $U_{\alpha}$ in the equations (27) and (43), respectively.

Based on these considerations, we choose as $U_{\alpha}$ the operator $U_{\alpha}^{(0)}$, action of which on the arbitrary function $\Psi$ on $r_{\alpha}, \Psi_{\beta}, x_{1}^{\prime}$ and $x_{2}^{\prime}$ is described by the relation

$U_{\alpha}^{(0)} \Psi=-\sum_{k=1,2}\left(\mu_{\beta k}\right)^{-1} \vec{\nabla}_{\vec{x}_{k}^{\prime}} \varphi_{\alpha} \vec{\nabla}_{\vec{x}_{k}^{\prime}}\left(\Psi / \varphi_{\alpha}\right)$.

After substituting (44) into (43), we obtain the equation for $\mathfrak{J}^{+}$, which can be conveniently written in the form:

$$
\left[E-E_{\alpha}+\frac{1}{2 \mu_{\beta}} \Delta_{\vec{r}_{\beta}}+\sum_{k=1,2}\left(\frac{1}{2 \mu_{\alpha k}} \Delta_{\vec{s}_{k}^{\prime}}+\frac{Z_{\alpha}}{s_{k}^{\prime}}\right)-\frac{Z_{\alpha} Z_{\beta}}{r_{\beta}}\right] \mathfrak{J}^{+}=0 .
$$

The solution of this equation is determined by comparing its asymptotics with the corresponding eikonal approximation. However, we will not further formalize these considerations. The technique required for this has been described in rather detail above when constructing a solution $\mathfrak{J}^{-}$of the similar equation (29). Lowering the rather cumbersome intermediate calculations, we write immediately the final result (at the limit $\left.m_{3,4}>m_{1}=m_{2}\right)$ :

$$
\begin{aligned}
& \mathfrak{I}^{+}=\mu_{\alpha}^{-2 i v_{\alpha}} N^{(-)^{*}}(v)\left[N^{(+)^{*}}\left(v_{\alpha}\right)\right]^{2} \exp \left(i \vec{k}_{\alpha} \vec{r}_{\alpha}\right), \\
& F\left(-i v, 1, i k_{\alpha} r_{\beta}+i \vec{k}_{\alpha} \vec{r}_{\beta}\right) \prod_{k=1}^{2} F\left(i v_{\alpha}, 1, i v s_{k}^{\prime}+i \overrightarrow{\mathrm{v}} \vec{s}_{k}^{\prime}\right),
\end{aligned}
$$

where $v_{\alpha, \beta}=Z_{\alpha, \beta} / v, v=Z_{\alpha} Z_{\beta} / v$.
Next, we need to substitute the expressions (23), (40), (42), (44), and (46) into the expression (16), after which the amplitude of reaction $T_{\alpha \beta}^{-}$can be written as the sum of two terms. This amplitude describes the simultaneous capture of two electrons by fast ions in collisions of with atoms:

$$
\begin{aligned}
& T_{\alpha \beta}^{-}=-\left[N^{(+)^{*}}\left(v_{\alpha}\right) N^{(+)^{*}}\left(v_{\beta}^{\prime}\right)\right]^{2} \times \\
& \times \iiint d \vec{x}_{1}^{\prime} d \vec{x}_{2}^{\prime} d \vec{r}_{\alpha} \exp \left(i \vec{k}_{\alpha} \vec{r}_{\alpha}+i \vec{k}_{\beta} \vec{r}_{\beta}\right) \Re\left(\vec{r}_{\alpha}, \vec{r}_{\beta}\right) \varphi_{\beta}^{*}\left(\vec{s}_{1}^{\prime}, \vec{s}_{2}^{\prime}\right) \times \\
& \times \prod_{j=1}^{2} F\left(i v_{\beta}^{\prime}, 1, i v^{\prime} x_{j}^{\prime}+i \vec{v}^{\prime} \vec{x}_{j}^{\prime}\right) \sum_{k=1,2} \vec{\nabla}_{\vec{x}_{k}^{\prime}} \varphi_{\alpha}\left(\vec{x}_{1}^{\prime}, \vec{x}_{2}^{\prime}\right) \vec{\nabla}_{\vec{s}_{k}^{\prime}} \times \\
& \times\left[F\left(i v_{\alpha}, 1, i v s_{1}^{\prime}+i \vec{v} \vec{s}_{1}^{\prime}\right) F\left(i v_{\alpha}, 1, i v s_{2}^{\prime}+i \vec{v} \vec{s}_{2}^{\prime}\right)\right]
\end{aligned}
$$

In the equation (47), the notation is used

$$
\begin{aligned}
& \mathfrak{R}\left(\vec{r}_{\alpha}, \vec{r}_{\beta}\right)=\mu_{\alpha}^{-2 i v_{\alpha}} \mu_{\alpha}^{-2 i v_{\beta}^{\prime}} N^{(-)^{*}}(v) N^{(-)^{*}}\left(v^{\prime}\right) \times \\
& \times F\left(-i v, 1, i k_{\alpha} r_{\beta}+i \vec{k}_{\alpha} \vec{r}_{\beta}\right) F\left(-i v^{\prime}, 1, i k_{\beta} r_{\alpha}+i \vec{k}_{\beta} \vec{r}_{\alpha}\right) .
\end{aligned}
$$

The obtained complex expression (47) is easily simplified. First of all, we note that in the case of rapid collisions (at $k_{\alpha}^{2} / 2 \mu_{\alpha}>>\left|E_{\beta}-E_{\alpha}\right|$ ), the incident particles are scattered mainly forward, i.e., at rather small angles $\hat{k}_{\beta} \cong \hat{k}_{\alpha}$. It is this region of scattering angles that makes a dominant contribution to the full cross-section of the reaction (2), since at large scattering angles the transition amplitude becomes small due to fast oscillations of the exponential factor $\exp \left(i \vec{k}_{\alpha} \vec{r}_{\alpha}+i \vec{k}_{\beta} \vec{r}_{\beta}\right)$ under the integral in (47). Physically this means that at small scattering angles, the trajectories can be considered almost straight and the nuclei move with a constant velocity vector. In this case, the vector $\vec{R}$ can be represented as an orthogonal sum $\vec{R}=\vec{\rho}+\vec{z}, \vec{\rho} \vec{z}=0$. Given that $\vec{v}^{\prime} \cong \vec{v}$ and $m_{1}=m_{2}=m<<m_{3} \approx m_{4}$ for the exponent in (47) it is easy to obtain the following relation:

$$
\vec{k}_{\alpha} \vec{r}_{\alpha}+\vec{k}_{\beta} \vec{r}_{\beta} \cong \vec{p}\left(\vec{x}_{1}+\vec{x}_{2}\right)+\vec{q}\left(\vec{s}_{1}+\vec{s}_{2}\right),
$$

where

$$
\begin{aligned}
& -2 \vec{p}=\vec{\eta}+\left(v-\frac{E_{\beta}-E_{\alpha}}{\mathrm{v}}\right) \hat{\mathrm{v}}, \\
& 2 \vec{q}=\vec{\eta}-\left(\mathrm{v}-\frac{E_{\beta}-E_{\alpha}}{\mathrm{v}}\right) \hat{\mathrm{v}} ; \quad \hat{\mathrm{v}}=\frac{\overrightarrow{\mathrm{v}}}{\mathrm{v}},
\end{aligned}
$$

$\vec{\eta}$ is orthogonal relatively to the vector $\vec{v}$ of the component of the vector of the transmitted momentum $(\vec{\eta} \vec{v}=\vec{\eta} \vec{z}=\vec{\rho} \vec{v}=\vec{\rho} \vec{z}=0)$.

For the function $\mathfrak{R}\left(\vec{r}_{\alpha}, \vec{r}_{\beta}\right)$ in this approximation, we get:

$$
\begin{aligned}
& \lim _{m_{\alpha, \beta} \rightarrow \infty} \mathfrak{R}\left(\vec{r}_{\alpha}, \vec{r}_{\beta}\right)=\mu^{-2 i\left(v_{\alpha}+v_{\beta}^{\prime}-v\right)}(\rho v)^{2 i v}, \\
& \mu=m_{\alpha} m_{\beta} /\left(m_{\alpha}+m_{\beta}\right) .
\end{aligned}
$$


Then, with the accuracy of the insignificant phase factor, expression for the scattering amplitude (i.e., charge-exchange (2)) at small angles has the form

$$
T_{\alpha \beta}^{-}=-\left[N^{(+)^{*}}\left(v_{\alpha}\right) N^{(+)^{*}}\left(v_{\beta}^{\prime}\right)\right]^{2} \sum_{k=1,2} I_{\alpha \beta}^{(k)},
$$

where the matrix elements are marked through $I_{\alpha \beta}^{(k)}$

$$
\begin{aligned}
& I_{\alpha \beta}^{(k)}=\iiint d \vec{x}_{1} d \vec{x}_{2} d \vec{s}_{1} \exp \left\{i \vec{p}\left(\vec{x}_{1}+\vec{x}_{2}\right)+i \vec{q}\left(\vec{s}_{1}+\vec{s}_{2}\right)\right\} \varphi_{\beta}^{*}\left(\vec{s}_{1}, \vec{s}_{2}\right) \times \\
& \times \prod_{j=1}^{2} F\left(i v_{\beta}, 1, i v x_{j}+i \vec{v} \vec{x}_{j}\right) \vec{\nabla}_{\vec{x}_{k}} \varphi_{\alpha}\left(\vec{x}_{1}, \vec{x}_{2}\right) \times \\
& \times \vec{\nabla}_{\vec{s}_{k}}\left[F\left(i v_{\alpha}, 1, i \cup s_{1}+i \overrightarrow{\mathrm{v}} \vec{s}_{1}\right) F\left(i v_{\beta}, 1, i \cup s_{2}+i \vec{v} \vec{s}_{2}\right)\right]
\end{aligned}
$$

We assume that the active electrons of system $\left(\lambda ; \gamma_{1}, \gamma_{2}\right), \lambda=\alpha, \beta$ move in the field of the atom residue and their motion is described by the Hamiltonian with separation of variables. Then the wave function $\varphi_{\alpha}\left(\vec{x}_{1}, \vec{x}_{2}\right)\left(\varphi_{\beta}\left(\vec{s}_{1}, \vec{s}_{2}\right)\right)$ of the initial (final) state can be represented as the product of the same (since, in the case of collision of a helium atom with $\alpha$-particle, the initial and final states are $S^{2}$-states) one-electron functions

$$
\begin{aligned}
& \varphi_{\alpha}\left(\vec{x}_{1}, \vec{x}_{2}\right)=\varphi_{\alpha}\left(\vec{x}_{1}\right) \varphi_{\alpha}\left(\vec{x}_{2}\right), \\
& \varphi_{\beta}\left(\vec{s}_{1}, \vec{s}_{2}\right)=\varphi_{\beta}\left(\vec{s}_{1}\right) \varphi_{\beta}\left(\vec{s}_{2}\right),
\end{aligned}
$$

where $\varphi_{\alpha}\left(\vec{x}_{k}\right)$ and $\varphi_{\beta}\left(\vec{s}_{k}\right)$ are hydrogen-like wave functions in the field of nuclei with effective charges $\alpha=Z_{\beta}-0.3125$ and $\beta=Z_{\alpha}-0.3125$ :

$$
\begin{aligned}
& \varphi_{\alpha}\left(\vec{x}_{k}\right)=\left(\alpha^{3} / \pi\right)^{1 / 2} \exp \left(-\alpha x_{k}\right), \\
& \varphi_{\beta}\left(\vec{s}_{k}\right)=\left(\beta^{3} / \pi\right)^{1 / 2} \exp \left(-\beta s_{k}\right) .
\end{aligned}
$$

We now begin to calculate the matrix element (53). Let us show how it can be done in the example of one of the terms in (52) by writing it in the momentum representation

$$
I_{\alpha \beta}^{(1)}=(2 \pi)^{-3} \int d \vec{\tau} \vec{R}_{\beta}^{(1)}(\vec{q}-\vec{\tau}) \vec{R}_{\alpha}^{(1)}(\vec{p}+\vec{\tau}) \vec{R}_{\beta}^{(2)}(\vec{q}+\vec{\tau}) \vec{R}_{\alpha}^{(2)}(\vec{p}-\vec{\tau}) .
$$

Here, we introduced the notations

$$
\vec{R}_{\beta}^{(j)}(\vec{k})=\int d \vec{s}_{j} \exp \left(i \vec{k} \vec{s}_{j}\right) \varphi_{\beta}^{*}\left(\vec{s}_{j}\right) \vec{\nabla}_{\vec{s}_{j}} F\left(i v_{\alpha}, 1, i v s_{j}+i \vec{v} \vec{s}_{j}\right),
$$

$\vec{R}_{\alpha}^{(j)}(\vec{k})=\int d \vec{x}_{j} \exp \left(i \vec{k} \vec{x}_{j}\right) \varphi_{\beta}\left(\vec{x}_{j}\right) F\left(i v_{\beta}, 1, i \cup x_{j}+i \vec{\cup} \vec{x}_{j}\right) \times$

$\times\left[\vec{\nabla}_{\vec{x}_{j}} \varphi_{\alpha}\left(\vec{x}_{j}\right)\right]$,

$$
R_{\beta}^{(j)}(\vec{k})=\int d \vec{s}_{j} \exp \left(i \vec{k} \vec{s}_{j}\right) \varphi_{\beta}^{*}\left(\vec{s}_{j}\right) F\left(i v_{\alpha}, 1, i \cup s_{j}+i \vec{\cup} \vec{s}_{j}\right),
$$

$R_{\alpha}^{(j)}(\vec{k})=\int d \vec{x}_{j} \exp \left(i \vec{k} \vec{x}_{j}\right) \varphi_{\alpha}\left(\vec{x}_{j}\right) F\left(i v_{\beta}, 1, i \cup x_{j}+i \vec{\cup} \vec{x}_{j}\right)$.
The expression under the integral (55) is localized in the following four regions of momentum $\vec{\tau}$ subspace:

$\begin{array}{ll}|\vec{p}-\vec{\tau}| \leq 1 / a, & || \vec{q}+\vec{\tau} \mid \leq 1 / a, \\ |\vec{p}+\vec{\tau}| \leq 1 / a, & |\vec{q}-\vec{\tau}| \leq 1 / a,\end{array}$

where $a$ is the characteristic radius of potentials of pair interaction. Since $\vec{R}_{\beta}^{(1)}(\vec{\tau})$ and $\vec{R}_{\alpha}^{(1)}(\vec{\tau})$ decrease faster than $\vec{R}_{\beta}^{(2)}(\vec{\tau})$ and $\vec{R}_{\alpha}^{(2)}(\vec{\tau})$, it is easy to understand that the contribution into the value of the integral (55) from the third and fourth areas in (60) is insignificant and its value is completely determined by the contribution of only the first and second areas in (60). Here, in the expression (55), the function $\vec{R}_{\beta}^{(1)}(\vec{q}-\vec{\tau}) \vec{R}_{\alpha}^{(1)}(\vec{p}+\vec{\tau}) \equiv R_{\alpha \beta}(\tau)$ can be taken outside the integral sign, which changes slowly in the areas of sharp growth of the rest of the integrand. Then applying the convolution theorem and performing the $\vec{x}$ integration according to the Nordsieck integral technique [16], we obtain

$$
\begin{aligned}
& I_{\alpha \beta}^{(1)}=-\frac{N_{\alpha} N_{\beta}}{2}\left[\vec{R}_{\beta}^{(1)}(\vec{q}-\vec{p}) \vec{R}_{\alpha}^{(1)}(2 \vec{p})+\vec{R}_{\beta}^{(1)}(2 \vec{q}) \vec{R}_{\alpha}^{(1)}(\vec{p}-\vec{q})\right] \times \\
& \times \frac{\partial}{\partial \alpha} \Gamma\left(\alpha+\beta, \vec{p}+\vec{q}, v_{\alpha}, v_{\beta}, \overrightarrow{\mathrm{v}}_{1}, \overrightarrow{\mathrm{v}}_{2}\right),
\end{aligned}
$$

where

$$
\begin{aligned}
& \Gamma\left(\lambda, \vec{k}, v_{1}, v_{2}, \overrightarrow{\mathrm{v}}_{1}, \overrightarrow{\mathrm{v}}_{2}\right)=\int d \vec{x} x^{-1} \exp (-\lambda x+i \vec{k} \vec{x}) \times \\
& \times F\left(i v_{1}, 1, i \mathrm{v}_{1} x+i \overrightarrow{\mathrm{v}}_{1} \vec{x}\right) F\left(i v_{2}, 1, i \mathrm{v}_{2} x+i \overrightarrow{\mathrm{v}}_{2} \vec{x}\right)= \\
& =4 \pi\left(k^{2}+\lambda^{2}\right)^{-1}\left[1+2\left(\vec{k} \overrightarrow{\mathrm{v}}_{1}-i \lambda \mathrm{v}_{1}\right) /\left(k^{2}+\lambda^{2}\right)\right]^{-i v_{1}} \times \\
& \times\left[1+2\left(\vec{k} \overrightarrow{\mathrm{v}}_{2}-i \lambda \mathrm{v}_{2}\right) /\left(k^{2}+\lambda^{2}\right)\right]^{-i v_{2}} F\left(i v_{1}, i v_{2}, 1, W\right), \\
& W=\frac{4\left(\vec{k} \overrightarrow{\mathrm{v}}_{1}-i \lambda \mathrm{v}_{1}\right)\left(\vec{k} \overrightarrow{\mathrm{v}}_{2}-i \lambda v_{2}\right)-2\left(\lambda^{2}+k^{2}\right)\left(\overrightarrow{\mathrm{v}}_{1} \overrightarrow{\mathrm{v}}_{2}-\mathrm{v}_{1} \mathrm{v}_{2}\right),}{\left[k^{2}+\lambda^{2}+2\left(\vec{k} \overrightarrow{\mathrm{v}}_{1}-i \lambda v_{1}\right)\right]\left[k^{2}+\lambda^{2}+2\left(\vec{k} \overrightarrow{\mathrm{v}}_{2}-i \lambda v_{2}\right)\right]} \\
& N_{\gamma}=\left(\frac{\gamma^{3}}{\pi}\right)^{1 / 2}, \gamma=\alpha, \beta .
\end{aligned}
$$

Interpretation of the expressions (61), (62) is as follows. The matrix element $\left(\vec{R}_{\beta}^{(1)} \vec{R}_{\alpha}^{(1)}\right)$ describes the two-step (Thomas') mechanism of electron capture $\left(\gamma_{1}\right)$ through the continuum from a target atom $\left(\beta ; \gamma_{1}, \gamma_{2}\right)$ to the states bound with the projectile $(\alpha)$. The factor $\partial \Gamma / \partial \alpha$, which corresponds to the integration in $I_{\alpha \beta}^{(1)}$ over the coordinates of second electron $\left(\gamma_{2}\right)$ is reduced to the overlap integral.

Similar in structure, the relations are obtained for the matrix element $I_{\alpha \beta}^{(2)}$. It can be seen that for the resonance two-electron capture, both matrix elements in (52) transform one into another when electrons transpose. Therefore, their contribution to the amplitude $T_{\alpha \beta}^{-}$is the same. 


\section{Results of calculations}

Let's consider the application of the above formalism on the example for the calculation of cross-sections of twoelectron charge-exchange in the collision of helium atom with $\alpha$-particles:

$\mathrm{He}^{2+}+\mathrm{He}\left(1 S^{2}\right) \rightarrow \mathrm{He}\left(1 S^{2}\right)+\mathrm{He}^{2+}$.

This process is important in connection with the problems of heating of deuterium-tritium plasma by $\alpha$ particles [17]. One way to diagnose $\alpha$-component of plasma is based on double charge-exchange (63) Megaelectron-Volts beams of helium atoms $\operatorname{He}\left(1 S^{2}\right)$ on fast $\alpha$-particles.

The process (63) was studied previously both theoretically $[18,19]$ and experimentally [20]. The most theoretical works $[18,19]$ use a model of independent events, in which charge exchange is interpreted as an event consisting of two independent events: one-electron - capture of one electron and "non-capture" of another one, two-electron - independent capture of both electrons. Because the probability of capturing an electron as a one-step process is determined by equality

$$
P(\vec{\rho})=\left|A_{\alpha \beta}^{(1)}(\vec{\rho})\right|^{2},
$$

where $A_{\alpha \beta}^{(1)}(\vec{\rho})$ is the amplitude of transition in the quasiclassical approximation, then the probability of a oneelectron charge-exchange is equal to $P^{2}$.

The correctness of using the model of independent events was discussed in [19], where for the reaction (63) at the collision energies $0.2-0.4 \mathrm{MeV} / \mathrm{a} . \mathrm{m} . \mathrm{u}$. the crosssections of two-electron capture were calculated. Double capture (63) is a two-electron process in which electronic correlations play a large role, so the limits of the model of independent electrons leads to differences between the calculated and experimentally measured cross-sections. Correlation effects affect both the value of cross-sections and the nature of their dependence on the collision energy [19].

In this relation, the correlated version of the Pluvinage correlated continuum distorted wave (PCCDW) approximation developed by Crothers and McCarroll [18] deserves some attention. In this approximation, electron capture is still an independent event, but electrons themselves are considered highly interacting in the entrance channel. The amplitude of single-electron capture in the formula (64) is determined in the standard CDW approximation [14], and the single-parameter correlated two-electron Pluvinage function is taken as the undisturbed wave function of the initial state [21].

In their model, Crothers and McCarroll believe that during a collision, electron is captured from the part of the target shell that is returned to the projectile. The second electron is inert and is located on the other side of the target due to the Pluvinage correlations.
Thus, the perturbation by the projectile potential before collision and the perturbation by the target potential after collision are sensed only by the captured electron. The undisturbed wave function of the finite state is represented as the product of two hydrogen orbitals.

The results of our calculations of cross-sections of resonance charge exchange (63) performed in the formalism of distorted waves with the amplitude (52) are presented in Fig. 1 (solid line) together with the results of the following authors' calculations: [22] - dashed, [23] dashed-dotted, [24] - short-dashed, [18] - short-dotted lines.

Fig. 2 shows the original results from the works: [20] - star, [22] - triangle, [25] - rhombus, which contain experimental data on cross-sections of double capture (63) at high energies.

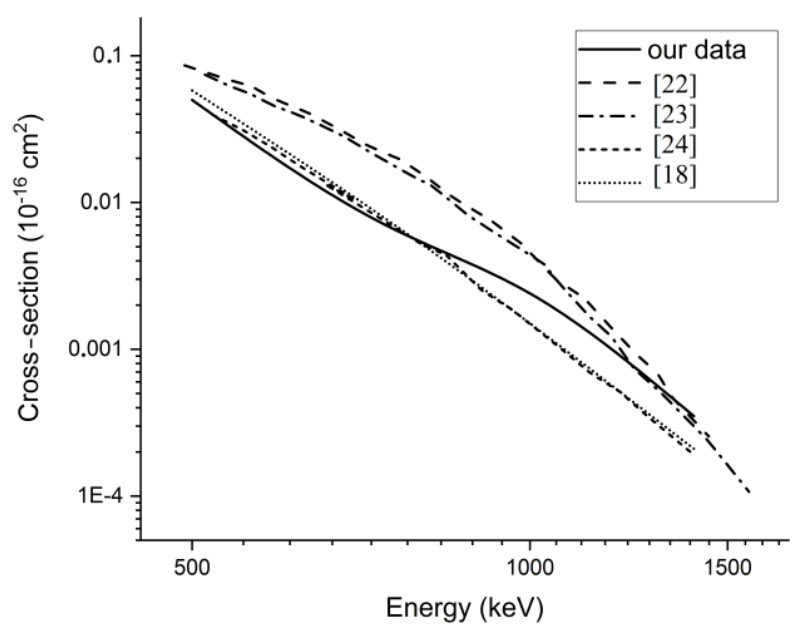

Fig. 1. The results of the calculations of the cross-sections of two-electron charge exchange during the collision of helium atom with $\alpha$-particles. Comparison with theoretical calculations.

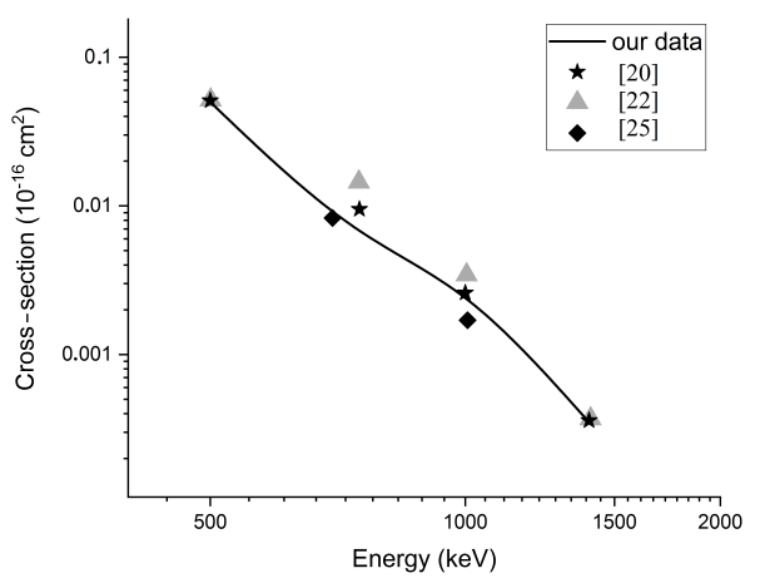

Fig. 2. Cross-section of two-electron charge exchange during collision of helium atom with $\alpha$-particles. Comparison with experimental data. 
The figures clearly show typical features of the calculation based on the use of the four-body Dodd-Greider integral equations in the calculation of amplitude, namely: a slight decrease in the calculated cross-sections in the area of average energies when comparing with the theoretical calculations of other authors and relatively good agreement with the experimental data at high energies. At the same time, as we see, the results of calculations in [22, 23] are overvalued not only with respect to our calculations and those of other authors [18, 24], but also with respect to the experiment [20]. This may be related with the fact that when the velocity of collision decreases, the approach of independent electrons becomes incorrect.

Fig. 1 also presents the results of the most accurate variants of the calculations performed by Crothers and McCarroll in the PCCDW approximation [18]. The data were obtained taking into account Coulomb distortions of electron wave functions in both reaction channels (63). We see that at $E=500 \mathrm{keV}$, the calculations [18, 24] are in good agreement with the experimental data. However, in the high energy area $(E \geq 1000 \mathrm{keV})$, the calculations made in $[18,24]$ lead to decreasing the cross-sections, and the proposed in this work method of accounting the Coulomb effects in the four-body model is better agreed with the experimental data.

\section{Conclusions}

On the basis of the Dodd-Greider integral equations for a quantum mechanical operator of a four-particle rearrangement scattering, the formalism of the continuum distorted wave method that is successfully used to describe two-electron capture processes in high-energy ion-atomic collisions has developed. The advantage of the proposed formalism over the others is a consistent preservation of the proper asymptotic limits of the wave functions of a colliding system in both channels of reaction, which takes into account the long-range nature of Coulomb interactions. The calculations of crosssections of double charge-exchange at the collision of $\mathrm{He}^{2+}$ with $\mathrm{He}$ were performed, which showed that the proposed method describes well the experimental data and the available theoretical calculations of other authors.

We have made the calculations on the assumption that the mechanism of the simultaneous capture of two electrons makes the major contribution to the amplitude of reaction (63) in the considered energy area. On the other hand, the charge-exchange processes in twoelectron systems can be considered as two-step processes of sequential capture of two electrons. Certainly, the simultaneous accounting the mechanisms mentioned above is a rather difficult task and requires further experimental and theoretical efforts. Further development of the method is seen in the accounting the additional mechanisms of two-electron capture, as well as in the inclusion of electronic correlations into the wave functions of the initial and final states. Obviously, these effects will be significant in the field of lower energies of the incident particles.

\section{References}

1. Lazur V.Yu., Khoma M.V. Distorted wave theories for one- and two-electron capture in fast atomic collisions. Advances in Quantum Chemistry. 2013. 65. P. 363-405. http://dx.doi.org/10.1016/b978-012-396455-7.00013-3.

2. Lazur V.Yu., Aleksiy V.V., Karbovanets M.I., Khoma M.V., Myhalyna S.I. Taking the Coulomb effects into account in the reactions of one-electron charge exchange. Semiconductor Physics, Quantum Electronics \& Optoelectronics. 2019. 22. P. 171181. https://doi.org/10.15407/spqeo22.02.171.

3. Belkić Dž. Quantum Theory of High-energy Ionatom Collisions. Taylor \& Francis, London, 2008.

4. Lazur V.Yu., Khoma M.V., Janev R.K. Asymptotic properties of the three-Coulomb-center problem eZ ZZ. Phys. Rev. A. 2006. 73. P. 032723. https://doi.org/10.1103/PhysRevA.73.032723.

5. Khoma M.V., Lazur V.Yu., Janev R.K. Asymptotic theory of the one- and two-electron processes in slow collisions of atomic ions with diatomic molecules. Phys. Rev. A. 2009. 80. P. 032706. https://doi.org/10.1103/PhysRevA.80.032706.

6. Belkić Dž. Total cross sections in five methods for two-electron capture by alpha particles from helium: CDW-4B, BDW-4B, BCIS-4B, CDW-EIS4B and CB1-4B. JOMC. 2020. 58. P. 1133-1176. https://doi.org/10.1007/s10910-020-01123-4.

7. Belkić Dž. Fast Ion-Atom and Ion-Molecule Collisions. World Scientific Publishing Co., Singapore, 2012. https://doi.org/10.1142/8485.

8. Fischetti M.V., Vandenberghe W.G. Advanced Physics of Electron Transport in Semiconductors and Nanostructures. Springer International Publishing, Switzerland, 2016.

9. Chao A., Chou W. Reviews of Accelerator Science and Technology - Vol. 10: The Future of Accelerators. World Scientific Publishing Co., Singapore, 2019.

10. Grozdanov T.P., Janev R.K., Lazur V.Yu. Asymptotic theory of the strongly asymmetric twoCoulomb-center problem. Phys. Rev. A. 1985. 32, No 6. P. 3425-3434. https://doi.org/10.1103/PhysRevA.32.3425.

11. Gorvat P.P., Lazur V.Yu. Asymptotic behavior of charge-exchange amplitude at relativistic velocities and binding energies. Theor. and Math. Phys. 1993. 95, No 3. P. 708-724. https://doi.org/10.1007/BF01017517.

12. Gorvat P.P., Lazur V.Yu., Presnyakov L.P., Uskov D.B. Asymptotic behavior of charge-exchange amplitude. Theor. and Math. Phys. 1992. 91, No 1. P. 373-384. https://doi.org/10.1007/BF01019830.

13. Lazur V.Yu., Mashika Yu.Yu., Janev R.K., Grozdanov T.P. Quasi-crossing of Rydberg terms in the problem of two Coulomb centers with strongly differing charges. Theor. and Math. Phys. 1991. 87, No 1. P. 401-410. https://doi.org/10.1007/BF01016580. 
14. Belkić Dž., Gayet R., Salin A. Electron capture in high-energy ion-atom collisions. Phys. Repts. 1979. 56, No 6. P. 279-369. https://doi.org/10.1016/03701573(79)90035-8.

15. Dodd L.R., Greider K.R. Rigorous solution of threebody scattering processes in the distorted-wave formalism. Phys. Rev. A. 1966. 146. P. 675-686. https://doi.org/10.1103/PhysRev.146.675.

16. Nordsieck A. Reduction of an integral in the theory of Bremsstranhlung. Phys. Rev. 1954. 93. P. 785787. https://doi.org/10.1103/PhysRev.93.785.

17. Barnett C.F., Harrison M.F.A. Plasmas: Applied Atomic Collision Physics. Academic Press, London, 1984.

18. Crothers D.S.F., McCarroll R. Correlated continuum distorted-wave resonant double electron capture in $\mathrm{He}^{2+}-\mathrm{He}$ collision. J. Phys. B. 1987. 20, No 12. P. 2835-2842. https://doi.org/10.1088/00223700/20/12/027.

19. Gayet R., Rivarola R.D., Salin A. Double electron capture by fast nuclei. J. Phys. B. 1981. 14, No 9. P. 2421-2427. https://doi.org/10.1088/00223700/20/12/027.

20. De Castro Faria N.V., Freire F.L. Jr., de Pinho A.G. Electron loss and capture by fast helium ions in noble gases. Phys. Rev. A. 1988. 37, No 1. P. 280283. https://doi.org/10.1103/PhysRevA.37.280.

21. Pluvinage $\mathrm{Ph}$. Fonction d'onde approchée à un paramètre pour l'état fondamental des atomes à deux électrons. Ann. Phys. (Paris). 1950. 12, No 5. P. 145-152.

https://doi.org/10.1051/anphys/195012050145.

22. Ghosh S., Dhara A., Mandal C.R., Purkait M. Double-electron-capture cross sections from helium by fully stripped projectile ions in intermediate-tohigh energies. Phys. Rev. A. 2008. 78. P. 042708. https://doi.org/10.1103/PhysRevA.78.042708.

23. Purkait M., Sounda S., Dhara A., Mandal C.R. Double-charge-transfer cross sections in inelastic collisions of bare ions with helium atoms. Phys. Rev. A. 2006. 74. P. 042723.

https://doi.org/10.1103/PhysRevA.74.042723.

24. Gravielle M.S., Miraglia J.E. Double-electron capture as a two-step process. Phys. Rev. A. 1992. 45, No 5. P. 2965-2973. https://doi.org/10.1103/PhysRevA.45.2965.

25. DuBois R.D. Double-electron capture as a two-step process. Phys. Rev. A. 1987. 36, No 6. P. 25852593. https://doi.org/10.1103/PhysRevA.36.2585.

\section{Authors and CV}

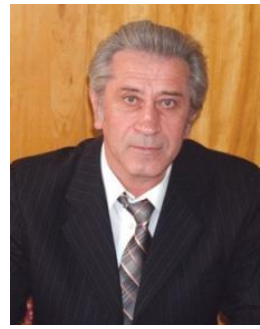

Volodymyr Yu. Lazur. Merited Figure of Science and Technology of Ukraine, Doctor of Physical and Mathematical Sciences, Leading Researcher, Professor of the Department of Theoretical Physics at the Uzhhorod National University, Ukraine. He is the author of more than 300 scientific publications. His main research interests of theoretical physics, theory of ion-atom and ion-molecular collisions. volodymyr.lazur@uzhnu.edu.ua

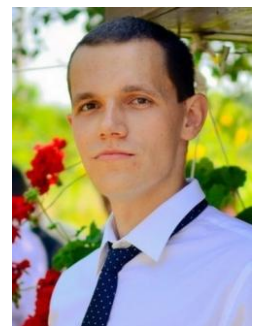

Vitaliy V. Aleksiy. Head of Laboratories of the Department of Theoretical Physics at the Uzhhorod National University, Ukraine. He is the author of about 20 scientific publications. The central research focus on theoretical physics, theory of ionatom and ion-molecular collisions.

vitaliy.aleksiy@uzhnu.edu.ua

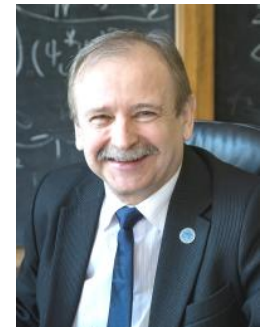

Michal Hnatič. Doctor Honoris Causa, Doctor of Sciences, Leading Scientist at the Institute of Expe-rimental Physics, Slovak Academy of Sciences, Professor of Depart-ment of Nuclear and Subnuclear Physics at P.J. Safarik University in Kosice, Slovak Republic. He is the author of more

than 180 scientific publications. The central researches have been focused on theoretical physics especially on quantum-field theory methods and their application in non-linear dynamics.

hnatic@saske.sk

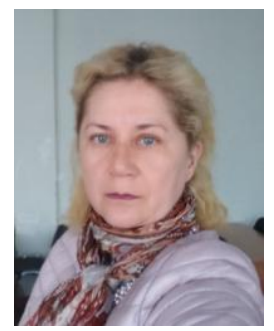

Svitlana I. Myhalyna. Senior Lecturer of the Department of Computer Systems and Networks at the Uzhhorod National University, Ukraine. She is the author of about 30 scientific publications. Her research areas are the theoretical physics and theory of ion-atom collisions.

kaf-networks@uzhnu.edu.ua 